\title{
The modulation of simple reaction time by the spatial probability of a visual stimulus
}

L.R.R. Carreiro,

H. Haddad Jr. and M.V.C. Baldo
Departamento de Fisiologia e Biofísica, Instituto de Ciências Biomédicas I, Universidade de São Paulo, São Paulo, SP, Brasil

\section{Correspondence \\ M.V.C. Baldo \\ Departamento de Fisiologia e \\ Biofísica, ICB I, USP \\ Av. Prof. Lineu Prestes, 1524 \\ 05508-900 São Paulo, SP \\ Brasil \\ Fax: +55-11-3091-7285 \\ E-mail: baldo@fisio.icb.usp.br}

Presented at the XVII Annual Meeting of the Federação de Sociedades de Biologia Experimental, Salvador, BA, Brazil, August 28-31, 2002.

Research supported by FAPESP (No. 96/06618-9). L.R.R. Carreiro was the recipient of a FAPESP fellowship (No. 99/07382-7).

Received April 12, 2002 Accepted February 25, 2003

\section{Abstract}

Simple reaction time (SRT) in response to visual stimuli can be influenced by many stimulus features. The speed and accuracy with which observers respond to a visual stimulus may be improved by prior knowledge about the stimulus location, which can be obtained by manipulating the spatial probability of the stimulus. However, when higher spatial probability is achieved by holding constant the stimulus location throughout successive trials, the resulting improvement in performance can also be due to local sensory facilitation caused by the recurrent spatial location of a visual target (position priming). The main objective of the present investigation was to quantitatively evaluate the modulation of SRT by the spatial probability structure of a visual stimulus. In two experiments the volunteers had to respond as quickly as possible to the visual target presented on a computer screen by pressing an optic key with the index finger of the dominant hand. Experiment $1(\mathrm{~N}=14)$ investigated how SRT changed as a function of both the different levels of spatial probability and the subject's explicit knowledge about the precise probability structure of visual stimulation. We found a gradual decrease in SRT with increasing spatial probability of a visual target regardless of the observer's previous knowledge concerning the spatial probability of the stimulus. Error rates, below $2 \%$, were independent of the spatial probability structure of the visual stimulus, suggesting the absence of a speed-accuracy trade-off. Experiment $2(\mathrm{~N}=12)$ examined whether changes in SRT in response to a spatially recurrent visual target might be accounted for simply by sensory and temporally local facilitation. The findings indicated that the decrease in SRT brought about by a spatially recurrent target was associated with its spatial predictability, and could not be accounted for solely in terms of sensory priming.
Simple reaction time (SRT) in response to visual stimuli has long been known to be influenced by several psychophysical features such as stimulus intensity (1-3), size $(4,5)$ and duration (6). Over the last several decades, many studies have also investigated
Key words

- Simple reaction time

- Spatial probability

- Vision

- Attention the influence of signal probability and statistical structure of signal sequences on human reaction time (7-10).

The speed and accuracy with which a human observer responds to a visual stimulus is affected by prior knowledge (higher 
predictability) about the stimulus location before its presentation. This is usually accomplished by means of peripheral or symbolic cues which indicate the probable location of a stimulus, generally reducing the reaction time with which an observer responds to it (11-14). However, manipulation of the spatial probability structure of the visual environment (15) can also be used to guide prior knowledge about stimulus location. This procedure might be a useful tool in charting the spatial distribution of visual attention, with no need for subsidiary stimuli such as peripheral or central cues.

The main purpose of the present study was to quantify the effect of a gradual shift in the spatial probability structure of a visual stimulus on human SRT (experiment 1). However, higher and lower probability locations are not comparable to each other with respect to the possibility of local sensory facilitation. The "repetition effect" is a phenomenon in which the average reaction time for a repeated signal is shorter than that for a new signal (16). Therefore, as a second goal, we also attempted to uncouple spatial predictability (here assumed to be a monotonic increasing function of spatial probability) and spatial recurrence, while holding constant the remaining psychophysical features of the visual stimuli (experiment 2).

Twenty-six volunteers gave informed consent to participate in these experiments, which were reviewed and approved by the Committee on Research Involving Human Subjects, Institute of Biomedical Sciences, University of São Paulo. All volunteers had normal or corrected-to-normal vision. They were seated in front of a computer monitor in a sound-attenuated, dimly lit room, and instructed to maintain stable eye position at a central fixation point during the task. A chin and forehead rest was used so that the distance from the observer's eyes to the screen was kept constant at $57 \mathrm{~cm}$. Eye position was monitored by a video camera.

During each trial, a fixation point was presented in the center of the screen. After a random interval (800-1800 ms), a target stimulus (a full square subtending $0.3^{\circ}$ of the visual angle with luminance of $21.4 \mathrm{~cd} / \mathrm{m}^{2}$ ) appeared at an eccentricity of $7.5^{\circ}$ either to the right or to the left of the fixation point. The observer had to respond as quickly as possible to the target presentation by pressing an optic key with the index finger of the dominant hand.

In experiment $1(\mathrm{~N}=14)$, the probability of a target to appear in the left or right hemifield was varied according to the following combination of right:left frequencies (predictability factor): 100:0, 90:10, 80:20, 70:30, 60:40, 50:50, 40:60, 30:70, 20:80, 10:90, and $0: 100 \%$. These combinations were run in separate, randomly ordered blocks under two conditions (expectancy factor): either with or without adding to the instructions previously given to the volunteer explicit information concerning the precise combination of frequencies to be employed.

Experiment $2(\mathrm{~N}=12)$ consisted of three conditions. Fixed condition: the target always appeared to the right or to the left of the fixation point depending on the experimental block. Alternate condition: the target location continuously alternated between the right and left hemifield throughout the block. Random condition: the target location (either right or left) was randomly chosen, with equal probability, from trial to trial. Whereas fixed and alternate conditions possess identical spatial predictabilities but different spatial and temporal features, the random condition exhibits, on average, the same spatial and temporal features assigned to the alternate condition, but a lower spatial predictability than both the fixed and alternate conditions.

The median SRT was calculated for each experimental condition and each volunteer separately. These values were entered into a multiway repeated-measure analysis of variance (ANOVA) followed by pairwise comparisons (Tukey HSD test), with the level of 
significance set at 5\%. In experiment 1 (Figure 1), whereas the effect of the predictability factor was a statistically significant reduction in the $\operatorname{SRT}[\mathrm{F}(9,117)=8.445$, $\mathrm{P}<0.0001]$, no main effect was observed for the expectancy factor $[\mathrm{F}(1,13)=0.018, \mathrm{P}=$ $0.893]$. In addition, we carried out an analysis of the errors observed in experiment 1 (anticipatory responses: SRT $<100 \mathrm{~ms}$; slow responses: SRT $>1000 \mathrm{~ms}$ ). Mean error rates related to anticipatory and slow responses were less than 1 and $2 \%$, respectively. None of these error rates was significantly influenced by either the predictability or the expectancy factor $(\mathrm{F}<1.381, \mathrm{P}>0.204)$.

In experiment 2 (Figure 2) a significant main effect $[\mathrm{F}(2,22)=6.0, \mathrm{P}=0.0084]$ was observed for the experimental condition (fixed, alternate and random). Pairwise comparisons revealed that the fixed and alternate conditions yielded mean SRT statistically indistinguishable from each other $(\mathrm{P}=0.835)$, and that these mean SRT were both significantly shorter than the mean SRT observed in the random condition ( $\mathrm{P}=0.036)$.

These findings indicate the strong influence of the spatial probability structure of the visual stimuli on simple reaction time. Response latencies gradually declined as the spatial probability of the stimulus was increased, and this effect did not depend on the previous information regarding the stimulus spatial probability. The volunteers seemed to acquire a subjective estimation on the basis of the probability structure of the visual signal, independent of the previous information concerning its specific statistical distribution. Therefore, if we accept that human observers are poor estimators of probability, we can conclude that previously informing the observer about the spatial probability structure of a visual stimulus was not an essential factor in defining the utility of a spatial location (15). The knowledge about the probability structure could then be experienced and assimilated during execution of the task, leading to an increase in the stimu- lus predictability and therefore improving performance, as measured by SRT. These findings are in agreement with results obtained in humans and monkeys by Ciaramitaro and colleagues (10).

These results also agree with previous studies in which stimulus probability was shown to modulate simple (8) and choice reaction time $(8,9)$ and visual search $(17)$, as well as visual sensitivity in humans and monkeys (10). Here we have expanded the range of spatial probabilities from 10 to $100 \%$ for SRT tasks (experiment 1), and devised an alternative way to determine whether a local sensory facilitation could fully account for the shortening of SRT with increasing stimulus probability (experiment 2). As a result of this second experiment, we found that the fixed condition (in which the stimulus was repeatedly presented at the same location throughout the session) yielded response latencies statistically indistinguishable from the response latencies generated under the alternate condition (in which the presentation time at a given location was, on average, identical to that generated under the random condition). In the random condition, the response latencies were significantly longer

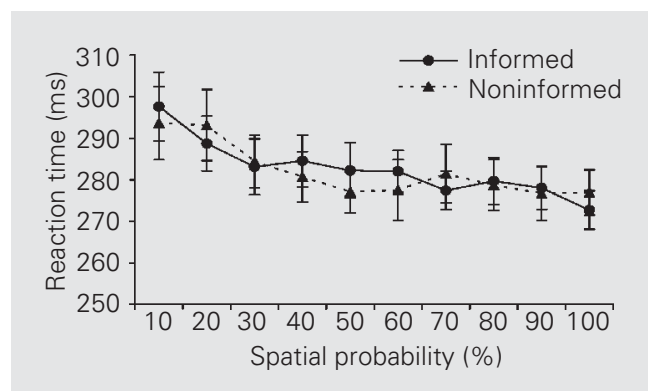

Figure 1. Mean reaction times as a function of spatial probability under informed and noninformed conditions (experiment 1). Data are reported as means \pm SEM for 14 subjects.

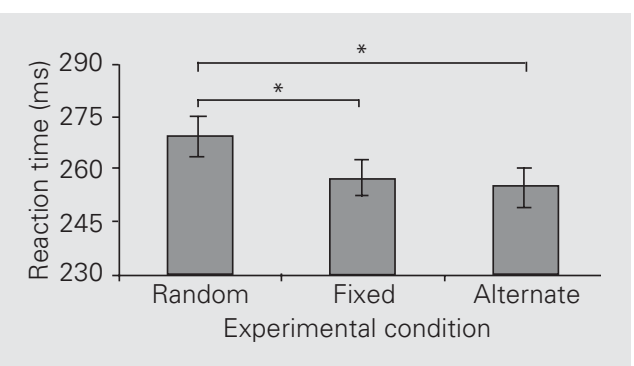

Figure 2. Mean reaction times for each experimental condition employed in experiment 2. Data are reported as means \pm SEM for 12 subjects. ${ }^{*} P<0.05$ for the comparisons indicated by the horizontal bars (Tukey HSD test). 
than those observed in both the fixed and alternate conditions, despite the fact that the random condition (characterized by a comparatively lower spatial probability) and the alternate condition shared the same statistical distribution of presentation times. Therefore, it seems unlikely that the reduction in SRT as a function of an increase in the stimulus spatial probability was simply due to a spatiotemporal sensory facilitation (sensory priming).

Moreover, if the reduction we observed in SRT with increasing predictability were due to a speed-accuracy trade-off, we should expect a dependence of the error rates (anticipatory and slow responses) on the spatial probability structure (namely, the predictability factor in experiment 1). Since this dependence was not observed, we are led to believe that the effect of spatial predictability on SRT might result from a change in the rate at which information is acquired rather than a change in the accuracy of the response (18). A further analysis, possibly by means of mathematical models of SRT, would be necessary to demonstrate the invariance of response bias with changes in the probability structure, thus strengthening the idea of a relationship between the rate of information acquisition and visual attention $(19,20)$. However, it would be premature to invoke an attentional mechanism in order to account for these findings. Generally, it is not an easy task to distinguish between genuine improvements in the quality of sensory signals (an expected result of attentional allocation) and mere changes in decisional criteria. This is particularly true in reaction time studies, in which a convincing role of attentional mechanisms usually requires the comparison between the performance of real observers and the performance of an ideal detector's model, such as the class of random walk models for reaction time (15). Nevertheless, our findings are consistent with previous work showing a reduction in response latencies as the result of an increase in the spatial predictability brought about by peripheral or symbolic cues (11-14). Although these reported findings are generally accepted as resulting from attentional mechanisms, we must be cautious in extending this interpretation to our present results.

It is conceivable that, by merely manipulating the probability structure of a visual stimulus regardless of explicitly informing the observer about that specific structure, the learned spatial predictability may yield the reallocation of visual attention, thus improving performance as revealed by SRT measures. If eventually accepted as a valid procedure to access the role of visual attention in SRT tasks, the manipulation of the probability structure of visual stimuli would allow us to map in greater detail the distribution of spatial attention over the visual field.

\section{Acknowledgments}

We thank Ronald Ranvaud and Luiz Ribeiro-do-Valle for valuable comments, Roberto Vieira for technical assistance, and an anonymous reviewer for helpful comments and suggestions.

\section{References}

1. Cattell JM (1886). The influence of the intensity of the stimulus on the length of the reaction time. Brain, 8: 512-515.

2. Piéron H (1920). Nouvelles recherches sur l'analyse du temps de latence sensorielle et sur la loi qui relie le temp a l'intensité d'excitation. Année Psychologique, 22: 58-142.

3. Ulrich R, Rinkenauer G \& Miller J (1998). Effects of stimulus duration and intensity on simple reaction time and response force. Journal of Experimental Psychology: Human Perception and Performance, 24:
915-928.

4. Dwyer W \& White CS (1974). Peripheral area-intensity interaction in simple visual reaction time. Vision Research, 14: 971-974.

5. Osaka N (1976). Reaction time as a function of peripheral retinal locus around fovea: effect of stimulus size. Perceptual and Motor Skills, 43: 603-606.

6. Ueno T (1978). Temporal summation in human vision: Simple reaction time measurements. Perception and Psychophysics, 23: 43-50. 
7. Hyman R (1953). Stimulus information as a determinant of reaction time. Journal of Experimental Psychology, 45: 188-196.

8. Krinchik EP (1969). The probability of a signal as a determinant of reaction time. In: Koster WG (Editor), Acta Psychologica 30 Attention and Performance II. North-Holland Publishing Company, Amsterdam, The Netherlands, 27-36.

9. Shaw ML (1978). A capacity allocation model for reaction time. Journal of Experimental Psychology: Human Perception and Performance, 4: 586-598.

10. Ciaramitaro VM, Cameron EL \& Glimcher PW (2001). Stimulus probability directs spatial attention: an enhancement of sensitivity in humans and monkeys. Vision Research, 41: 57-65.

11. Posner MI, Snyder CRR \& Davidson BJ (1980). Attention and the detection of signals. Journal of Experimental Psychology: General, 109: 160-174.

12. Riggio L \& Kirsner K (1997). The relationship between central cues and peripheral cues in covert visual orientation. Perception and Psychophysics, 59: 885-899.

13. Turatto M, Benso F, Facoetti A, Galfano G, Mascetti GG \& Umiltà C (2000). Automatic and voluntary focusing of attention. Perception and Psychophysics, 62: 935-952.

14. Handy TC, Green V, Mangun RG \& Klein RM (2001). Combined expectancies: Event-related potentials reveal the early benefits of spatial attention that are obscured by reaction time measures. Journal of Experimental Psychology: Human Perception and Performance, 27: 303-317.

15. Sperling G \& Dosher BA (1986). Strategy and optimization in human information processing. In: Boff K, Kaufman L \& Thomas J (Editors), Handbook of Perception and Performance. Vol. 1. Wiley, New York, 2.1-2.65.

16. Bertelson P (1961). Sequential redundancy and speed in a serial two-choice responding task. Quarterly Journal of Experimental Psychology, 12: 90-102.

17. Shaw ML \& Shaw P (1977). Optimal allocation of cognitive resources to spatial locations. Journal of Experimental Psychology: Human Perception and Performance, 3: 201-211.

18. Link SW (1975). The relative judgment theory of two-choice response time. Journal of Mathematical Psychology, 12: 114-135.

19. Carrasco M \& McElree B (2001). Covert attention accelerates the rate of visual information processing. Proceedings of the National Academy of Sciences, USA, 98: 5363-5367.

20. Cameron EL, Tai JC \& Carrasco M (2002). Covert attention affects the psychometric function of contrast sensitivity. Vision Research, 42: 949-967. 